\title{
EXTREMAL LENGTHS ON DENJOY DOMAINS
}

\author{
R. C. PENNER
}

(Communicated by Irwin Kra)

\begin{abstract}
We consider the problem of computing the extremal lengths of certain homotopy classes of curves in certain symmetric surfaces. Specifically, we concentrate on plane domains which are conformal to the Riemann sphere with a collection of slits in the real axis removed; such a conformal type is called a Denjoy domain. Using Jenkins-Strebel forms, the extremal length of any sufficiently symmetric homotopy class of curves is computed in terms of the endpoints of the slits. One can then choose a symmetric pants decomposition of the surface and invert the formulas derived, which are a set of coupled quadratic equations. In this way, one obtains a coordinatization of the space of all marked Denjoy domains of a fixed topological type.
\end{abstract}

We consider the (marked) conformal type of a domain of the form $F=\$^{2}-\Sigma$, where $\boldsymbol{S}^{2}$ denotes the Riemann sphere, and $\Sigma \subset \boldsymbol{S}^{1} \subset \boldsymbol{S}^{2}$ consists of a finite collection of arcs, called slits. The extremal length (defined below) is a number associated with a conformal class of metric and a homotopy class of connected curves in $F$. In this note, we prove an analogue of the Fricke-Klein Theorem to the effect that extremal lengths of a finite family of simple curve classes uniquely determine conformal type. (This is in contrast to [HM], where homotopy classes of families of curves are considered.) This "injectivity question" on a general Riemann surface has been considered in [Pi], where the analogue of the Fricke-Klein Theorem is proved for the sphere-minus-four-points.

The author would like to thank the staff and members of the Institut MittagLeffler for their hospitality during 1983-1984, when this work was done. Particular thanks are due to Allen Weitsman for several helpful discussions.

Let $F^{r}=S^{2}-\left\{\bigcup_{j=1}^{r-1}(2 j-3,2 j-2) \cup(2 r-3, \infty)\right\}$ and let $\Delta_{i}$ be a simple closed curve encircling the $i$ th component of $\mathbb{S}^{2}-F^{r}, i=1, \ldots, r$, as in Figure 1. A pants decomposition $P$ of $F^{r}$ is a collection of simple curves $\left\{\Gamma_{j}\right\}_{2}^{r-2}$ (as in Figure 1) so that components of $F-\bigcup\left\{\Gamma_{j}\right\}$ have Euler characteristic -1 .

A metric $\mu$ on $F^{r}$ will be regarded as a line element $d s=f(z)|d z+g(z) d \bar{z}|$ where $f \geq 0$ with isolated zeros and $\|g\|_{\infty}<1$. The Teichmüller space $\tau^{r}$ of $F^{r}$ is the space of conformal classes of finite-area complete metrics on $F^{r}$ modulo pushforward by diffeomorphisms isotopic to Dehn twists along $\Delta_{i}, i=1, \ldots, r$. We take $r \geq 3$ so that $F^{r}$ is equipped with the intrinsic Poincaré metric $\mu_{\rho} \in \rho \in \tau^{r}$ (see $[\mathbf{A b}])$.

Let $\iota: F^{r} \eta$ denote the involutive orientation-reversing homeomorphism induced by conjugation on $S^{2}$ and $\iota^{*}: \tau^{r} \eta$ the corresponding homeomorphism. A conformal class $\rho \in \tau^{r}$ is said to be a (marked) Denjoy domain (and we write

Received by the editors June 11, 1986 and, in revised form, December 16, 1986.

1980 Mathematics Subject Classification (1985 Revision). Primary 30CXX, 30FXX; Secondary 30C75, 30C20, 30F 15. 


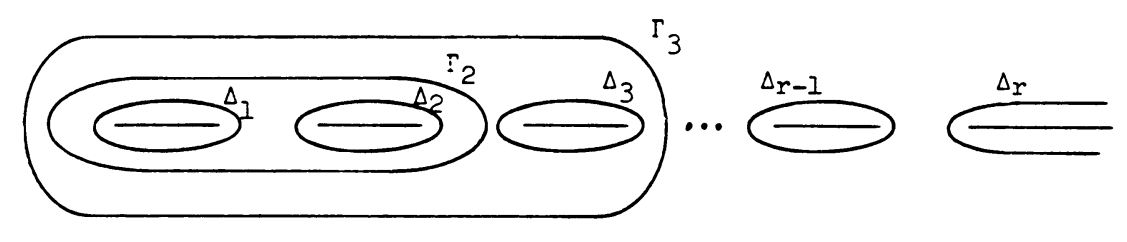

FIGURE 1

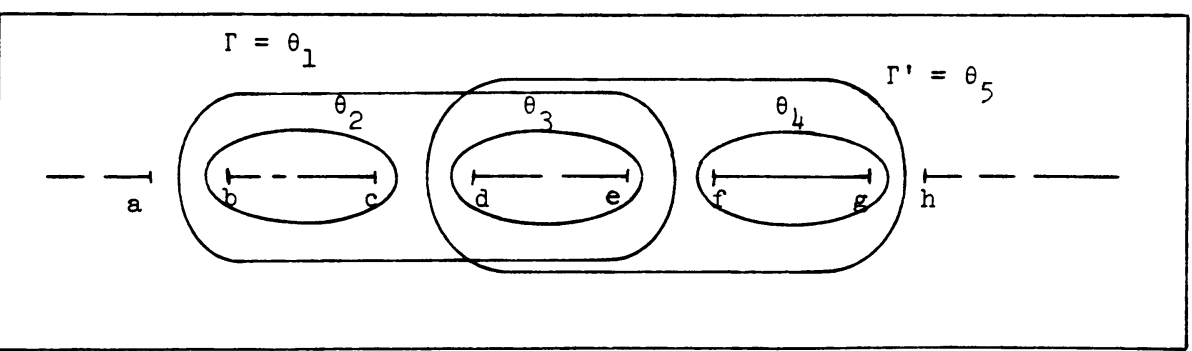

FIGURE 2

$\left.\rho \in D^{r} \subset T^{r}\right)$ if $\iota^{*} \rho=\rho$. $\left(D^{r}\right.$ is the real Teichmüller space of type $F^{r} / \iota$ in the sense of [Ea].) A pants decomposition $P$ is symmetric if $\iota P$ is isotopic to $P$.

Suppose $\Gamma$ is a homotopy class of simple curve in $F^{r}$ and $\mu$ is a metric on $F$. We let $\mu(\Gamma)=\inf _{\gamma \in \Gamma}(\mu$-length of $\gamma$ ) and define the extremal length (see $[\mathbf{A h}])$ of $\Gamma$ for $\rho \in \tau^{r}$ to be

$$
\lambda_{\rho}(\Gamma)=\sup _{\mu \in \rho} \frac{[\mu(\Gamma)]^{2}}{\left(\mu \text {-area of } F^{r}\right)} .
$$

Our main result follows.

THEOREM. Suppose $P=\left\{\Gamma_{j}\right\}_{2}^{r-2}$ is a symmetric pants decomposition. The map

$$
\begin{aligned}
\Lambda & : D^{r} \rightarrow \mathbf{R}_{+}^{2 r-3} \\
& : \rho \rightarrow\left(\lambda_{\rho}\left(\Delta_{i}\right)\right)_{1}^{r-1} \times\left(\lambda_{\rho}\left(\Gamma_{j}\right)\right)_{2}^{r-2}
\end{aligned}
$$

is a proper homeomorphism.

It is standard [HM] that $\Lambda$ is continuous on $\tau^{r}$, and insofar as $\mu_{\rho}(\Gamma) \leq \pi \lambda_{\rho}(\Gamma)$, Mumford's [Mu] exhaustion of $\tau^{r}$ shows that $\Lambda$ is proper. Our work involves showing $\Lambda$ is bijective.

The link between conformal geometry and topology is provided by quadratic differentials (see $[\mathbf{J e}, \mathbf{S t}])$. We recall the

JENKINS-STREBEL THEOREM. Given an isotopy class $\Gamma$ of simple curves on $F^{r}$ and $\rho \in \tau^{r}$, there is a unique projective class of holomorphic quadratic differentials $\omega=\omega_{\rho}(\Gamma)$ so that all the noncritical trajectories of $\omega$ lie in $\Gamma$.

The metric of $\omega$ is the extremal metric for $\lambda_{\rho}(\Gamma)$. The union of the noncritical trajectories of $\omega$ forms an annulus $A_{\rho}(\Gamma)$ whose modulus is the reciprocal of $\lambda_{\rho}(\Gamma)$. 
We will also need the Grötsch function (see [LV, p. 53])

$$
\begin{aligned}
& \mu:(0,1) \rightarrow \mathbf{R}_{+} \\
& \quad: r \rightarrow \text { modulus of } \mathbf{D}^{2}-(0, r),
\end{aligned}
$$

where $\mathbf{D}^{2}$ denotes the unit disc in $\boldsymbol{S}^{2}$. The key fact [Ah] for us is that $\mu$ is a homeomorphism. We define the Teichmüller annulus $T(a)=\mathbb{S}^{2}-((-1,0) \cup$ $(a, \infty)), a \in \mathbf{R}_{+}$, and recall [LV, Theorem II. 1.1] that the modulus of $T(a)$ is $2 \mu\left((1+a)^{-1 / 2}\right)$. The Teichmüller width $x_{\rho}(\Gamma)$ is the value of $a \in \mathbb{R}_{+}$so that $T(a)$ is conformal to $A_{\rho}(\Gamma)$. It follows that

$$
1+x_{\rho}(\Gamma)=\left[\mu^{-1}\left(1 /\left(2 \lambda_{\rho}(\Gamma)\right)\right)\right]^{-2} .
$$

The correspondence between Teichmüller widths and extremal lengths is seen to be bijective, and the former will prove more natural for what follows.

Given a sequence $0<a_{1}<\cdots<a_{2 r-3}$ of positive reals, we define the Riemann surface $F\left(a_{1}, \ldots, a_{2 r-3}\right)$ to be $\boldsymbol{S}^{2}-\bigcup_{1}^{r}\left(a_{2 j-3}, a_{2 j-2}\right)$ equipped with the spherical metric, where we have set $a_{-1}=-1, a_{0}=0, a_{2 r-2}=\infty$ for convenience. It follows from the Riemann mapping theorem that if $\rho \in D^{r}$, then there is an orientation-preserving conformal homeomorphism $\left(F^{r}, \rho\right) \rightarrow F=F\left(a_{1}, \ldots, a_{2 r-3}\right)$, which preserves marking in the obvious sense. We identify homotopy classes of curves on $F^{r}$ with those in $F$, and let $\iota: F$ ? denote the restriction of conjugation on $\mathbb{S}^{2}$ to $F$.

LEMMA. The theorem holds if $P$ is the standard pants decomposition illustrated in Figure 1.

ProOF. Suppose that $\rho \in D^{r}$ is conformally equivalent to $F=F\left(a_{1}, \ldots, a_{2 r-3}\right)$, $0<a_{1}<\cdots<a_{2 r-3}$. Let

$$
\begin{array}{rlrl}
x_{i} & =x_{\rho}\left(\Delta_{i}\right), & & i=1, \ldots, r, \\
y_{j}=x_{\rho}\left(\Gamma_{j}\right), & i=2, \ldots, r-2 .
\end{array}
$$

We claim that the following equalities hold.

$$
\begin{aligned}
x_{1} & =a_{1}, \\
x_{i} & =\frac{\left(a_{2 i-1}-a_{2 i-2}\right)\left(a_{2 i-3}-a_{2 i-4}\right)}{\left(a_{2 i-1}-a_{2 i-4}\right)\left(a_{2 i-2}-a_{2 i-3}\right)}, \quad i=2, \ldots, r-1, \\
x_{r} & =\frac{a_{2 r-3}-a_{2 r-4}}{1+a_{2 r-4}}, \\
y_{j} & =\frac{a_{2 j-1}-a_{2 j-2}}{1+a_{2 j-2}}, \quad j=2, \ldots, r-2,
\end{aligned}
$$

where we have again set $a_{0}=0$ for convenience.

We start with the first equation and consider the ring domain $A=A_{\rho}(\mu)$ in $F$. Since $A$ is invariant under $\iota$, so too is the critical locus $Z$ of the Jenkins-Strebel form $\omega_{\rho}(\Gamma)$. Suppose that $\gamma \subset Z$ is a piecewise analytic arc with endpoints in $S^{1}=\partial \mathbf{D}^{2}$. If $\gamma \not \subset \mathbb{S}^{1}-\bigcup_{1}^{r}\left(a_{2 j-3}, a_{2 j-2}\right)$, then there is a subarc $\gamma^{\prime} \subset \gamma$ with $\gamma^{\prime} \cap \mathbb{S}^{1}=\partial \gamma^{\prime} \cap \mathbb{S}^{1}$ and $\gamma^{\prime} \cup \iota\left(\gamma^{\prime}\right)$ separates $S^{2}$. This is a contradiction since $F-Z=A$ is connected. Thus $Z \subset S^{1}$, so $A$ is the Teichmüller annulus $T\left(a_{1}\right)$, whence $x_{1}=a_{1}$, as was claimed. Routine computations with Möbius transformations similarly give the other equations $(*)$. 
We prove the Lemma by showing that the coupled system of $2 r-3$ nonlinear equations (*) defines a bijection between $\left\{0<a_{1}<\cdots<a_{2 r-3}<\infty\right\}$ and $\mathbf{R}_{+}^{2 r-3}$. This involves computing $a_{k}$ inductively for $k=1, \ldots, 2 r-3$ from $\left(x_{i}\right) \times\left(y_{j}\right)$, checking at each step existence (independent of $\left(x_{i}\right) \times\left(y_{j}\right)$ ) and uniqueness of a solution with $a_{k}>a_{k-1}, k=1, \ldots, 2 r-3$, and the case $k=1$ is trivial.

The cases $k=2,3$ on $F^{3}$ will be considered separately first. The equation for $x_{3}$ gives that $a_{3}=x_{3}+a_{2}\left(1+x_{3}\right)$, and the equation for $x_{2}$ gives the quadratic

$$
\begin{aligned}
0 & =x_{2}\left(1+x_{3}\right) a_{2}^{2}+\left(x_{2} x_{3}-x_{1} x_{2}-x_{1} x_{3}-x_{1} x_{2} x_{3}\right) a_{2}-x_{1} x_{3}\left(1+x_{2}\right) \\
& =\alpha a_{2}^{2}+\beta a_{2}+\gamma
\end{aligned}
$$

in $a_{2}$. Now, $\alpha>0$ and $\gamma<0$, so there is a unique positive solution if and only if the discriminant is positive. One checks that this is automatic for $x_{1}, x_{2}, x_{3}>0$ and that $0<a_{1}<a_{2}<a_{3}<\infty$, and the Lemma follows in this case.

If $r>3$, suppose that $0<a_{1}<\cdots<a_{2 K-3}$ are known and the corresponding $x_{1}, \ldots, x_{2 K-3}$ are unrestricted. We introduce the change of variables

$$
a=a_{2 K-4}, \quad b=a_{2 K-3}-a_{2 K-4}, \quad c=a_{2 K-2}-a_{2 K-4}, \quad d=a_{2 K-1}-a_{2 K-4},
$$

and the equations for $x_{K}$ and $y_{K}$ yield the quadratic

$$
\begin{aligned}
0 & =x_{K}\left(1+y_{K}\right) c^{2}+\left[x_{K} y_{K}(1+a)-b\left(1+y_{K}\right)-b y_{K}\right] c-b y_{K}(1+a)\left(1+x_{K}\right) \\
& =\alpha c^{2}+\beta c+\gamma .
\end{aligned}
$$

Again $\alpha>0, \gamma<0, \beta^{2}>4 \alpha \gamma$ hold automatically and $0<c<d$.

Finally, the computation of $a_{2 r-4}$ and $a_{2 r-3}$ parallels the computation of $a_{2}$ and $a_{3}$ on $F^{3}$ above, and the Lemma follows.

We define a move on a symmetric pants decomposition $P$ as follows. Suppose $\Gamma \in P$ and $\Gamma^{\prime}$ is a $\iota$-invariant curve class disjoint from $P-\Gamma$ with $\Gamma^{\prime} \cap \Gamma=2$ as in Figure 2. We say the symmetric pants decomposition $P^{\prime}=P \cup \Gamma^{\prime}-\Gamma$ arises from $P$ by an exchange. An easy argument shows that exchanges act transitively on symmetric pants decompositions.

Suppose $P^{\prime}$ differs from $P$ by an exchange and assume the theorem for $P$. Adopt the notation of Figure 2 and normalize so that $a=\infty, b=-1$, and $c=0$. Computations already done in the Lemma allow one to compute $\left(x_{\rho}\left(\theta_{i}\right)\right)_{2}^{5}$ uniquely from $\left(x_{\rho}\left(\theta_{i}\right)\right)_{1}^{4}$, and the theorem follows.

We have proved somewhat more than the theorem. The transformation on Teichmüller widths is $\mathbf{R}$-algebraic (in the sense that its graph is a $\mathbf{R}$-algebraic variety intersected with the positive orthant). It follows that the centralizer of $\iota$ in $\pi_{0}$ Homeo $F^{r}$ acts R-algebraically on our "Teichmüller width coordinates" on $D^{r}$. Moreover, we have explicitly computed Teichmüller widths of symmetric curves in terms of endpoints $a_{1}, \ldots, a_{2 r-3}$.

\section{REFERENCES}

[Ab] W. Abikoff, The real analytic theory of Teichmüller space, Lecture Notes in Math., vol. 280, Springer-Verlag, Berlin and New York, 1980.

[Ah] L. V. Ahlfors, Conformal invariants, McGraw-Hill, New York, 1973.

[Ea] C. J. Earle, The moduli space of a closed Riemann surface with symmetries, Advances in the Theory of Riemann Surfaces, Ann. of Math. Stud., no. 66, Princeton Univ. Press, Princeton, N. J., 1971, pp. 119-130. 
[HM] J. H. Hubbard and H. Masur, Quadratic differentials and foliations, Acta Math. 142 (1979), 221-274.

[Je] J. A. Jenkins, On the existence of certain general extremal metrics, Ann. of Math. 66 (1957), 440-453.

[Mu] D. Mumford, A remark on Mahler's compactness theorem, Proc. Amer. Math. Soc. 28 (1971), 289-294.

[Pi] E. Pitz, Extremal length and Teichmüller theory, thesis, Techn. Univ., Berlin, 1983.

[Lv] O. Lehto and K. I. Virtanen, Quasiconformal mappings in the plane, Springer-Verlag, Berlin and New York, 1973.

[St] K. Strebel, Quadratic differentials, Springer-Verlag, Berlin and New York, 1984.

Department of MATHEMAtics, University of Southern California, Los ANGELES, CALIFORNIA 90089 